\title{
Technologies for Highly Parallel Optoelectronic Integrated Circuits
}

\author{
Kevin L. Lear \\ Sandia National Laboratories \\ Photonics Research Department \\ MS 0603 / P. O. Box 5800 \\ Albuquerque, NM 87185-0603
}

\begin{abstract}
Market considerations motivate optoelectronic integrated circuits for highly parallel intrasystem optical buses. Surface normal device technologies are well suited for these applications. System examples highlight device technology issues.
\end{abstract}

\section{INTRODUCTION}

Researchers in optoelectronics face the challenge of developing semiconductor devices and systems which manipulate photons with as much dexterity and economy as offered by transistors and integrated circuits in controlling electrons. Monolithic integration of optoelectronic elements is accepted as a key requirement to establishing such a technology.

Analogous to electronic ICs, both performance and manufacturing issues motivate optoelectronic integrated circuits (OEICs). Vertical integration, i.e. the monolithic combination of different electronic and optical components, enhances circuit functionality and reduces parasitic elements associated with hybrid circuit interconnection. Reduced discrete component counts ease manufacturing. Even more, this factor drives increasingly large arrays of single types of optoelectronic components, i.e. horizontal integration, for parallel optical operations. The most sophisticated OEICs include both vertical and horizontal integration. Optoelectronic devices are evolving from discrete components to forms appropriate for these various types of integration.

While summarily reviewing the range of OEICs, this paper emphasizes technology for highly parallel optical interconnections. Market volume and integration suitability considerations highlight board-to-board interconnects within systems as an initial insertion point for large OEIC production. The large channel count of these intrasystem interconnects necessitates two-dimensional laser transmitter and photoreceiver arrays. Surface normal optoelectronic components are promoted as a basis for OEICs in this application. An example system is discussed that uses vertical cavity surface emitting lasers for optical buses between layers of stacked multichip modules. Another potentially important application for highly parallel OEICs is optical routing or packet switching, and examples of such systems based on smart pixels are presented.

\section{OEIC MARKETS}

Computation and communication are the two major applications areas for modern microelectronics. Optical devices are far more useful in the latter and unlikely to compete with electronic devices in the former unless new technologies are developed in the long term. In the broadest sense, optical communications includes any form of transmitting information in an optical signal. In addition to primary markets such as general purpose data communications and telecommunications, this view includes encoding optical carriers with microwave phase information for distribution to segmented antennas or multiple channel frequencies for cable television. These modulation applications require more sophisticated optoelectronics, but do not rival the volume of digital communications markets.

Optoelectonics applications in sensing, including spectroscopy, ranging, guidance, and detection, as well as medical applications can also benefit from OEICs. The ability to integrate sources, detectors, and analysis devices such as interferometers or spectrometers on a chip is particularly attractive for portable or dedicated field instruments. Potential medical applications range from diagnostic equipment such as blood glucose monitors to exotic plans for laser tweezers and flow cytometry chips. Sensing applications are growing, and some passive optical chips are produced today for interferometry and gyroscopes. However, this market is unlikely to require large levels of integration at the volume expected for communications.

Generally, the number of physical optical communications links and hence optoelectronic transmitters and receivers increases as the length of the link decreases. There are more trunk lines than transoceanic ones. Anticipating the insertion of optoelectronics more deeply into systems, there will need to be more channels for board-to-board interconnects in computers than for local area networks. Large volume, low cost markets more strongly motivate the manufacturing advantages of monolithic integration and justify the overhead costs of the requisite technology development. Conversely, high cost, low volume markets can more readily support the additional per unit costs of hybrid assembly. Therefore short, intrasystem communications is likely to lead the push for large volume optoelectronic integration.

Some factors moderate the certainty of this conclusion. Success requires both a shift from electronic to optical interconnection as well as the concurrent development of appropriate OEICs. A slowing in the pace of system speed increases or dramatic improvements in electronic interconnects would inhibit the OEIC market for interconnects. This would shift the emphasis to the intersystem link OEIC market where simple optical channels have already been inserted. In part, the success of the larger potential market for intrasystem OEICs will depend on the demand for advanced systems. 


\section{DISCLAIMER}

Portions of this document may be illegible in electronic image products. Images are produced from the best available original document. 
An important exception to the inverse market volume versus communication distance relationship may occur if all optical networks become reality. In these networks, switches and not just transceivers would be based on optoelectronic devices. Since there are more switches than transceivers by a factor of at least $\log _{2} \mathrm{~N}$ in an $\mathrm{N}$-input system, such a change from electronic to optical switches could produce a sizable market in addition to the transceivers that are anticipated to dominate in the near future.

Not only does the number of links increase as the link length decreases, but the physical channel count per link also tends to increase, and the data transmission rates fall. Expensive transceivers employing sophisticated multiplexing schemes are appropriate for more fully utilizing the large bandwidth of long links with high cabling costs. However, the transceiver cost dominates the transport media cost for short hauls, promoting simple optoelectronic designs. Clock recovery, framing, error correction, auto leveling circuitry, and especially multiplexing several electronic signals onto a few optical channels are not cost effective for board to board interconnects. Multiplexers in particular require faster technology than the base system technology implying higher cost and greater power dissipation.

This leads to intrasystem interconnects being highly parallel, effectively employing spatial multiplexing rather than time or wavelength division multiplexing. From the electronic perspective this amounts to one-for-one replacement of bus wires with optical beams constituting an optical backplane. Since the optical signals aren't multiplexed, the required bandwidth corresponds to the system clock frequencies, typically much lower than the optical channel capabilities. This provides a large margin for future increases in the electronic system speed. Clearly horizontal integration, i.e. the fabrication of large monolithic arrays of simple optoelectronic transmitters and receivers, is most pertinent for short interconnects or optical backplanes while vertical integration is more important for sophisticated long

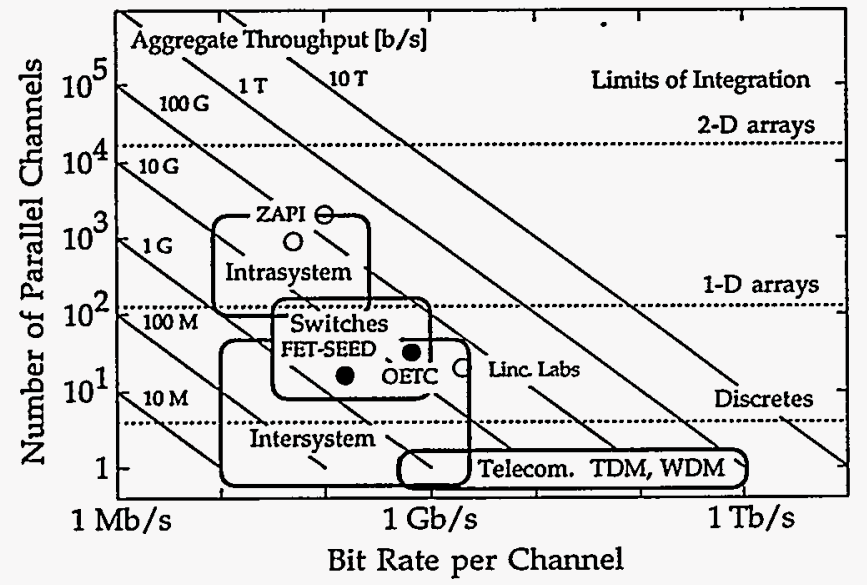

Figure 1. (After figure from N. K. Dutta.) Regimes for different optical communications markets are shown as a function of speed and channel count along with points for specific systems that have been demonstrated (solid dot) or are in progress (open circle) which are discussed in section VII. Approximate limits for varying dimensions of integrated arrays are also indicated. haul links. Applications requiring large, simple arrays are a particularly attractive arena for promoting OEIC manufacturing infrastructure. An established horizontal integration capability can then gracefully evolve to more sophisticated and specialized vertical integration technology.

Figure 1 presents a map of optical communications systems with respect to the coordinates of channel count and individual channel bandwidth. Aggregate system bandwidth contours are also shown. Presently, the frontier for technology development is in the multi-Gb/s realm for the extremes of single channel long haul telecommunications and highly parallel board-to-board interconnects as well as parallel links for computer local area networks and packet switching systems that fall in between. Different integrable device technologies are competing for being the foundation for OEICs to be incorporated into these systems. Issues such as speed, parallelism, and signal complexity of the systems form the basis for selecting a particular technology.

\section{OEIC CLASSIFICATIONS}

One distinction used to classify technologies is the role of electronics in OEICs. Many optical chips include electronic devices because of their versatility. Chips that maintain a continuous optical signal path from input to output are distinguished as photonic integrated circuits (PICs). The remainder employ devices such as lasers and light emitting diodes to convert electronic signals to optical ones and photodetectors for the reciprocal process. The term optoelectronic integrated circuit (OEIC) most strictly applies to those chips that have mixed electronic and optical signals although it is sometimes also used to refer to refer to a broader class that includes PICs. Electronic devices often provide gain or perform logic or signal processing in OEICs while optical signals are used for either the input or output or both. Within PICs, optical amplifiers can provide gain, and switching can be accomplished by various types of couplers, interferometers, and absorbers.

PICs are well suited for applications where high speed requirements or sophisticated signal modulation (usually analog) favor maintaining an optical signal format. Optical modulators have been reported at frequencies up to $40 \mathrm{GHz}$ [1], which is faster than laser diodes can be modulated. More importantly they produce less chirp than laser diodes. PICs may incorporate optical heterodyning for shifting carriers down to intermediate frequencies that are compatible with electronics.

Some switching may also be best accomplished by PICs. In particular, optical switching is attractive if there is excessive overhead in converting from optical to electrical signals and back again and if sophisticated logical processing of the signal is not required. In fact, optical switching may be better suited to communication formats that establish connections for the duration of the session than for packet switching where the packet header information must be reprocessed at each step and switches are reconfigured for each packet. 
In addition to classifying optical ICs according to signal medium, they can also be categorized according to physical geometry. The two types are waveguiding where light is transmitted from device to device in the plane of the wafer or surface normal where light is transmitted perpendicular to the wafer plane. In the waveguiding geometry both components as well as signal paths are defined on the chip. In the surface normal geometry the chip serves as a platform for components, but the light path is externally defined.

\section{WAVEGUIDE PICS}

Typically, PICs are based on waveguides because they require more extensive manipulation of the light. This follows from the need to combine multiple devices or stages to process the signal optically. Light is most conveniently routed between devices on the chip using waveguides rather than using off-chip optics. Beyond simple routing, waveguides can be used for optical signal combining or splitting. Again this would require either off-chip optics or sophisticated binary optics in the surface normal case. Additionally, modulator technology is central to optical signal manipulation, and waveguide modulators are more mature than surface normal ones.

There are other consequences of light propagation in the plane of the wafer that affect the utility of waveguide devices. Waveguide geometries offer long interaction lengths with epitaxial layers. This can lead to high quantum efficiency detectors or large phase shift modulators. However, devices may need to be quite long for such performance, leading to large chip sizes. In part, this is a consequence of active region layers being much thinner than the vertical extent of the optical beam in the waveguide. If the light is more tightly squeezed into the active region by altering waveguide design parameters, the far field diffraction angle increases complicating coupling. Optical coupling is a major issue for waveguides. The small waveguide apertures especially complicate coupling optical beams propagating in free space. Thus waveguiding optical ICs usually are directly coupled to sources, detectors, or fibers.

Surface normal coupling into and out of planar waveguide devices could substantially improve the utility of these components in applications that use free space beams such as board-to-board interconnects. It might also ease the packaging and alignment constraints for coupling into fiber optics for longer haul applications. Either diffraction gratings[2] or turning mirrors can redirect the waveguide beam perpendicular to the surface. Beam astigmatism can also be corrected by sophisticated versions of these optical components such as parabolic mirrors[3] or anamorphic binary lenses. Without surface normal emission, planar waveguide chips are limited to one-dimensional arrays which aren't dense enough for high channel count applications. Creating the gratings or mirrors for surface normal operation that enables two-dimensional arrays requires very sophisticated etching or lithography capabilities.

\section{SURFACE NORMAL COMPONENTS}

Surface normal components, and thus dense, twodimensional arrays, can be realized with conventional microelectronics fabrication tools if optical propagation is perpendicular to the wafer within the optical layers. Twodimensional arrays are important for the short haul, high channel count systems which are potentially the largest volume market for OEICs as discussed earlier. Device technology that fosters horizontal integration for simple parallel optical transmitters and receivers should retain conventional fabrication to promote manufacturing. The surface normal geometry also allows large symmetric apertures for simplified coupling to either free space beams or fiber optics. Surface normal configurations do sacrifice on chip signal routing unless holographic gratings for substrate mode connections [4] or comparably complicated features are introduced with the attendant difficulties in fabrication.

The primary difficulty with surface normal components is the short interaction length with thin active layers. A method that has been used to overcome this limitation for lasers and modulators is to enhance the interaction by placing the active layers in a high Q-factor resonant optical cavity. The mirrors for the cavity are made from quarterwave distributed Bragg reflectors usually comprising $\mathbf{3 0}$ to 80 epitaxial layers. The mirror layers and cavity must have very accurate thicknesses to establish the proper resonance condition. This structure is crucial for a type of laser diode called the vertical cavity surface emitting laser (VCSEL). These lasers use only a few quantum wells in order to obtain low operating currents. Their performance is summarized below. Another cavity device, the asymmetric Fabry-Perot modulator[5] can modulate the reflection, transmission, or phase of an input optical beam based on spoiling or shifting the cavity resonance.

Not all surface normal components rely on optical cavities. Absorption modulators, such as the self-electrooptic effect (SEED) device[6], can also be made from large numbers of quantum wells without requiring a cavity. Since the device absorbs rather than emits light, the operating current is modest even for a large number of layers. Excellent direct bandgap photodetectors can also be made with only a few microns of epitaxy and don't require cavities. Resonant cavity detectors may be useful, however, for strained layer material systems with limited critical layer thicknesses and for silicon or other indirect materials with low absorption coefficients.

\section{LASER TECHNOLOGIES FOR PARALLEL INTERCONNECTS}

Laser diode geometries have evolved in order to be compatible with monolithic integration. Traditionally, semiconductor lasers are based on planar waveguides bounded by mirrors formed from cleaved facets. This technology restricts laser arrays to one-dimensional bars with typical widths of $1 \mathrm{~mm}$ or less, complicating handling issues. Packaging tolerances are also tight since the light is emitted from the edge of the chip in close proximity to any 
supporting structure or heatsinking. Typical, inexpensive discrete lasers of this type are abundant byproducts of the compact disk player industry.

Substantial improvements in the power dissipation of edgeemitting lasers have resulted in arrays appropriate for parallel optical interconnects. Reductions in size, incorporation of strained quantum wells, and more sophisticated processing and growth has reduced threshold currents by two orders of magnitude to values as low as $0.2 \mathrm{~mA}$ [7]. This also permits lasers to be driven to many times threshold directly from digital circuits, avoiding complicated laser drivers.

Vertical cavity surface emitting lasers (VCSELs) [8] also offer submillampere threshold currents while affording many other benefits, chiefly being well suited for two-dimensional integrated laser arrays. Since the demanding task of forming mirrors is done through epitaxy, fabrication is simplified and must only laterally delineate the laser. Lasers can be patterned side-by-side in large arrays [9] as shown in Figure 2. Some optoelectronic component manufacturers feel that VCSELs can be made at the same cost as surface emitting LEDs once the complicated epitaxial structure can be grown repeatably.

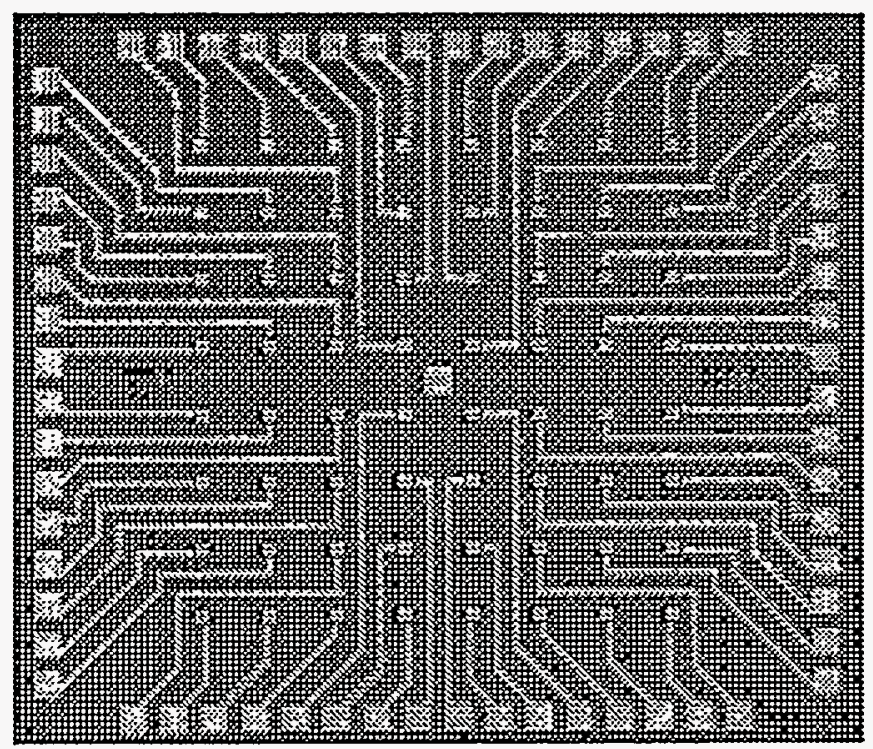

Figure 2. Photograph of an $8 \times 8$ VCSEL array. Dice such as this one will be used in board-to-board interconnects. The laser pitch is $250 \mu \mathrm{m}$.

The VCSEL's ultra-short cavity and surface normal geometry result in important optical properties. The large, symmetric laser cross-section gives circular low divergence beams. They support only one longitudinal mode and either one or more lateral modes depending upon size and drive level. The laser beam is emitted either out of the surface of the chip or down through the substrate, directions that can simplify packaging.

VCSELs are mostly still in the research and development phases, but are rapidly progressing towards being practical devices. Early devices had large resistances due to mirror layer heterojunctions, resulting in voltages at threshold of several volts. The corresponding high power dissipation rapidly overheated the devices, limiting output power. These problems have been greatly reduced by mirror grading[10] or alternative injection schemes[11]. Operating voltages are now only a few tenths of a volt higher than for edge-emitting lasers. This is particularly important for compatibility with direct drive from low supply voltage circuits. VCSELs can now produce a few milliwatts of single-mode power and tens of milliwatts in multi-mode operation. Published reliability studies are scarce, but preliminary results indicate no significant problems[12]. Both large corporations and small specialized companies expect to commercialize VCSEL products within a few years.

The potential for two-dimensional laser arrays have lead to research on alternatives to VCSELs. A class of devices called in plane surface emitting lasers use conventional laser waveguide structures, but deflect the output beams with mirrors or gratings as discussed earlier. Such devices have not received as much research support as VCSELs and lag behind them in maturity.

\section{ARRAY OEIC SYSTEM EXAMPLES}

One-dimensional and two-dimensional laser arrays and receiver OEICs are being used in systems demonstrations for both board-to-board interconnects as well as intersystem links. These systems examples highlight the present research technology for both edge-emitting and surface-emitting laser arrays as well as modulators.

Workers at Lincoln Laboratories and Rockwell are currently developing a board-to-board optical interconnect using an array of low threshold edge-emitting lasers stood on end[13]. The $980 \mathrm{~nm}$ lasers are directly driven from ECL circuits. The receiver uses InGaAs p-i-n diodes flip-chip mounted on a three stage AlGaAs heterojunction bipolar transistor detector and amplifier circuit. A 20 channel interconnect has been operated up to $3 \mathrm{~Gb} / \mathrm{s}$ per channel as indicated in Figure 1 . Even lower threshold laser arrays with integral control electrodes have been developed at the University of Southern California. Initial fiber optic data link experiments have been conducted at $1 \mathrm{~Gb} / \mathrm{s}$ using these devices[14].

The anticipated manufacturing advantages of VCSELs over edge-emitting lasers has motivated multichannel fiber optic links based on one-dimensional VCSEL arrays. One such intersystem link is a project of the Optoelectronics Technology Consortium(OETC). It has produced a 34 channel parallel link operating near $500 \mathrm{Mb} / \mathrm{s}$. This system is represented in Figure 1.

An example of a system incorporating two-dimensional VCSEL arrays is the Z-axis photonic interconnect (ZAPI) project at Sandia[15]. The project is developing technology for optical signal buses between layers of stacked multichip modules (MCMs). This is effectively a board-to-board interconnect in a very tightly packaged system where the boards are MCMs. While the connections between MCM layers are optical, the connections between chips on each layer are conventional electrical ones. One target system would be based on $50-100 \mathrm{Mb} / \mathrm{s} \mathrm{CMOS}$ and require 2000 optical channels per layer operating at this rate. This combination, which is consistent with the Semiconductor 
Industry Association road map projections for the year 2001, amounts to an aggregate bandwidth of $100 \mathrm{~Gb} / \mathrm{s}$ as plotted in Figure 1. In order to meet a power budget of $20 \mathrm{~W}$ per layer for the optical interconnects, the combined power consumption of the laser driver, laser, and receiver circuit for each channel would be only $10 \mathrm{~mW}$.

Several approaches were evaluated for meeting these demanding requirements for bandwidth and power consumption. Long electrical lines routed through vias and button connectors at the module edges encounter difficulties with these combinations of speeds and densities. As pitches decrease below $1 \mathrm{~mm}$, crosstalk and line parasitics begin to affect electrical interconnect speed. The relative advantages of optical interconnects will increase as speeds, line lengths, or channel counts increase. Given optical connections, multiplexing would allow fewer physical optical channels, but the additional overhead in terms of power and transceiver circuit complexity make this approach unworkable. For the unmultiplexed approach that was chosen, driver and receiver circuits must be minimized. The VCSELs will be directly driven from CMOS, and the receiver circuit will be based on a $\mathrm{p}-\mathrm{i}-\mathrm{n}$ photodiode and single heterojunction bipolar transistor stage.

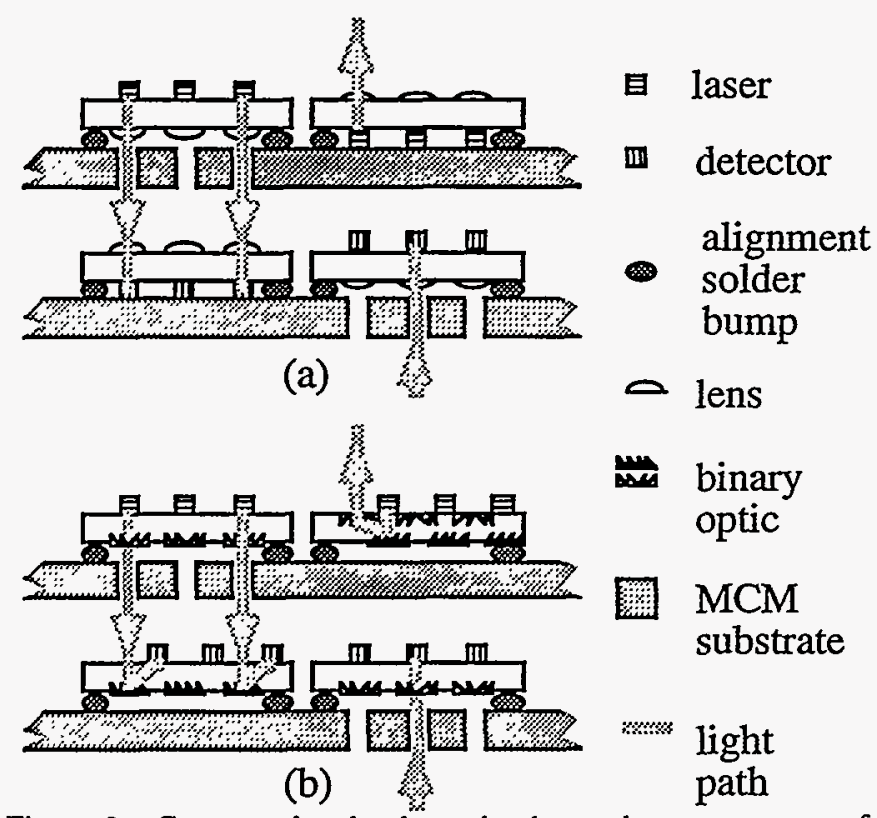

Figure 3. Cross-sectional schematic shows the arrangement of laser and receiver array chips for Sandia's ZAPI project which optically interconnects layers of stacked MCMs. One configuration (a) needs only simple lenses for beam shaping but requires mixed chip mounting. A more sophisticated approach (b) relies on sophisticated binary optics to permit all chips to be mounted face up.

The ZAPI project requires advances in photonic packaging. A schematic cross-section of the stacked MCM is shown for two different chip configurations in Figure 3 High array densities and small detector areas require precision alignment between layers. Optical vias must be laser drilled to allow transmission of $980 \mathrm{~nm}$ laser light through the silicon MCM. In the future $1300 \mathrm{~nm}$ light could pass through the silicon without a via, but suitable VCSELs have not yet been demonstrated at this wavelength. Lenses are formed on the back of the optoelectronic die to collimate the beams between layers. For the configuration shown in Figure 3(a), the bottoms of the optoelectronic chips always face each other so simple lenses will suffice. However, this configuration requires a mix of flip chip and normal mounting of the lasers and detectors. In order to be compatible with some MCM approaches, such as the high density interconnect, all of the electrical connections must be made on the top side of the die. This either requires vias in the flipped chips or, as shown in Figure 3(b), more complicated reflective and transmissive binary optics.

VCSEL based arrays are being monolithically combined with detectors and electronics for yet more sophisticated applications. Such array cells that receive and transmit optical signals as well as processing them are called smart pixels. One smart pixel system based on monolithically integrated VCSELs, photodiodes, and heterojunction bipolar transistors is being developed for optical routing[16]. This optical switching fabric uses bypass-exchange switches which are programmed indirectly by optical inputs processed through electronics. Non-planarity issues have to be addressed in the fabrication of OEICs such as this one that stack the epitaxial layers for devices on top of each other. One solution is to incorporate regrowth to position the devices side-by-side.

An alternative smart pixel technology based on monolithically integrating SEED modulators and FETs has been used to demonstrate a $155 \mathrm{Mb} / \mathrm{s} 32 \times 16$ free space optical switching fabric[17]. While most PICs are based on waveguides, the earlier definitions qualify this system as a surface normal PIC. The operation requires only one interaction with a modulator per chip but substantial external optics for signal routing from chip to chip through five stages.

\section{CONCLUSIONS}

These systems require substantial further development before being practical. This in turn relies on advances in OEICs. Large, surface normal laser transmitter and receiver arrays are particularly attractive vehicles for building OEIC technology. Maintaining simplicity in these components will assist in their insertion into applications. A potentially large market for highly parallel intrasystem interconnects is well matched to this approach. The success of volume OEIC production for this application will depend upon future system requirements and technical advances in electronic interconnects. Other markets that may strongly motivate OEICs are intersystem links and all optical networks. Waveguiding geometries can meet the reduced channel count requirements in these applications.

\section{ACKNOWLEDGMENT}

I gratefully acknowledge R. Carson and C. Sullivan for useful discussions. This work was supported by the United States Department of Energy under Contract DE-AC0494AL85000. 


\section{REFERENCES}

[1] R. G. Walker, "High-Speed III-V Semiconductor Intensity Modulators," IEEE J. Quant. Elec. , vol. 27, pp. 654--667, 1991.

[2] S. H. Kravitz, G. R. Hadley, M. E. Warren, J. R. Wendt, G. A. Vawter, J. C. Word, R. F. Corless, R. F. Carson, M. G. Armendariz and B. E. Hammons, "Waveguide-to-Fiber Coupling Using a Second-Order Grating and an Anamorphic Binary Optic," IEEE Lasers and Electro-Optics Society 1993 Annual Meeting, vol. 6, pp. 472--473, San Jose, CA: 1993.

[3] J. P. Donnelly, W. D. Goodhue, C. A. Wang, R. J. Bailey, G. A. Lincoln, G. D. Johnson, L. J. Missaggia and J. N. Walpole, "CW Operation of Monolithic Arrays of SurfaceEmitting AlGaAs Diode Lasers with Dry-Etched Vertical Facets and Parabolic Deflecting Mirrors," Photon. Tech. Lett. , vol. 5, pp. 1146--1149, 1993.

[4] R. K. Kostuk, J. W. Goodman and L. Hesselink, "Optical Imaging Applied to Microelectronic Chip-to-Chip Interconnections," Appl. Opt. , vol. 24, p. 2851, 1985.

[5] I. J. Fritz, J. A. Olsen, A. J. Howard, T. M. Brennan, B. E. Hammons and G. A. Vawter, "Strained-Layer-Superlattice Technology for Vertical-Cavity Optoelectronic Modulators at Near-Infrared Wavelengths," IEEE J. Quant. Elec. , vol. 30, pp. 452--458, 1994.

[6] L. A. D'Asaro, L. M. F. Chirovsky, E. J. Laskowski, S. S. Pei, T. K. Woodward, A. L. Lentine, R. E. Leibenguth, M. W. Focht, J. M. Freund, G. G. Guth and L. E. Smith, "Batch Fabrication and Operation of GaAs-AIGaAs Field-Effect Transistor-Self-Electrooptic Effect Device (FET-SEED) Smart Pixel Arrays," IEEE J. Quant. Elec. , vol. 29, pp. 670--677, 1993.

[7] H. Zhao, M. H. MacDougal, N. C. Frateschi, S. Siala, P. D. Dapkus and R. N. Nottenburg, "High Efficiency InGaAs/GaAs Single-Quantum-Well Lasers Using SingleStep Metalorganic Chemical Vapor Deposition," Photon. Tech. Lett. , vol. 6, pp. 468--470, 1994.

[8] P. L. Gourley, K. L. Lear and J. Schneider Richard P., "Surface-emitting lasers," IEEE Spectrum, vol. 31, pp. 31-37, August 1994.

[9] D. Vakhshoori, J. D. Wynn, G. J. Zydzik and R. E. Leibenguth, "8 X 18 top emitting independently addressable surface emitting laser arrays with uniform threshold current and low threshold voltage," Appl. Phys. Lett. , vol, 62, pp. 1718--1720, 1993.

[10] K. L. Lear, R. P. Schneider, K. D. Choquette, S. P. Kilcoyne, J. J. Figiel and J. C. Zolper, "Vertical Cavity Surface Emitting Lasers with $21 \%$ Efficiency by Metalorganic Vapor Phase Epitaxy," Photon. Tech. Lett., vol. 6, no. 9, 1994.

[11] A. Scherer, J. L. Jewell, M. Walther, J. P. Harbison and L. T. Florez, "Fabrication of Low Threshold Voltage Microlasers," Electron. Lett. , vol. 28, pp. 1224--1226, 1992.

[12] D. Vakhshoori, J. D. Wynn, R. E. Leibenguth and R. A. Novotny, "Long lasting vertical-cavity surface-emitting lasers," Electron. Lett. , vol. 29, pp. 2118--2119, 1993.

[13] D. Tsang, private communication.

[14] S. Siala, H. Zhao, M. Govindarajan, R. N. Nottenburg and P. D. Dapkus, "Synchronous DC-coupled parallel optical data path using three-terminal InGaAs/GaAs lasers," Electron. Lett. , vol. 30, pp. 1165--1166, 1994.
[15] R. F. Carson, P. K. Seigal, D. C. Craft and M. L. Lovejoy, "Future Manufacturing Techniques for Stacked MCM Interconnections," Journal of Metals, vol. pp. 51--55, June 1994.

[16] J. Cheng, P. Zhou, S. Z. Sun, S. Hersee, D. R. Myers, J. Zolper and G. A. Vawter, "Surface-Emitting Laser-Based Smart Pixels for Two-Dimensional Optical Logic and Reconfigurable Optical Interconnections," IEEE J. Quant. Elec. , vol. 29, pp. 741--756, 1993.

[17] F. B. McCormick, T. J. Cloonan, A. L. Lentine, J. M. Sasian, R. L. Morrison, R. A. Novotny, M. G. Beckman, S. L. Walker, M. J. Wojcik, S. J. Hinterlong, R. J. Crisci and H. S. Hinton, "Smart pixel-based switching fabric demonstration," Tech. Digest of Conference on Lasers and Electro-Optics 1994 , vol. 8, pp. 373--374, Anaheim, CA: 1994.

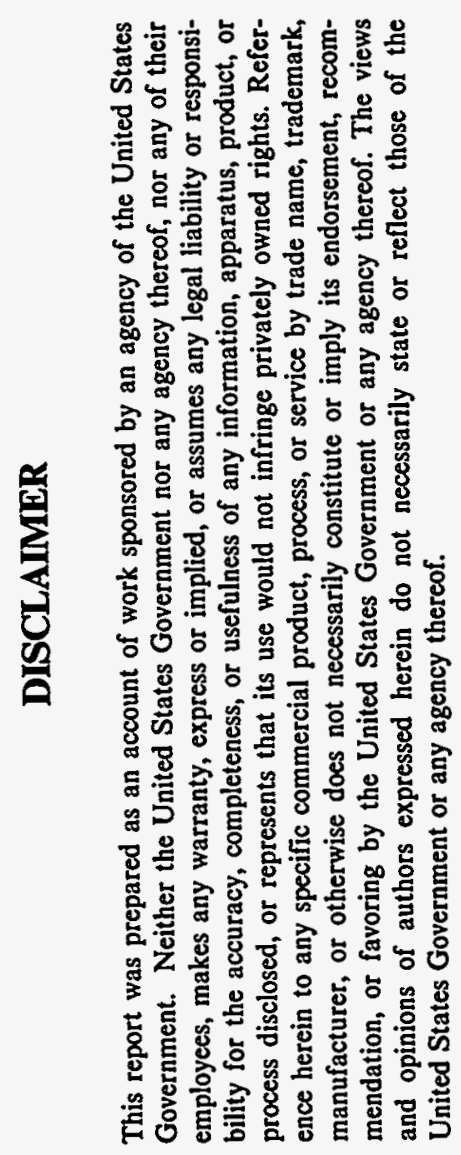

